\title{
What is the cost of implementing updated NICE guidance (2018) on venous thromboembolism prophylaxis post hospital discharge for medical patients?
}

We are writing regarding our experience of attempting to calculate the costs and benefits of implementing the recommendation by the National Institute for Health and Care Excellence (NICE) for 7 days prophylactic low-molecular weight heparin $(\mathrm{LMWH})$ in medical patients deemed at high risk of venous thromboembolism (VTE), which has caused recent debate. ${ }^{1-4}$

In 2018, at our trust, 1,230 medical patients aged $\geq 85$ years old were admitted for 3-6 days. Using the Department of Health and Social Care's VTE risk assessment tool, medical patients admitted with a decrease in baseline mobility for more than 3 days and $>60$ years old would require 7 days prophylactic LMWH (we are aware that the majority of medical patients get prophylactic LMWH). We assumed that any patient $<85$ years old would be able to inject LMWH and patients admitted for less than 3 days would not need ongoing prophylactic LMWH after discharge. We collected data on patients aged $\geq 75$ years old, including a frailty score based on the Clinical Frailty Scale. ${ }^{5}$ Locally, $80 \%$ of patients aged $\geq 85$ years old counted as frail (a score $\geq 5$ ). We assumed that frail patients needed prophylactic LMWH injecting on their behalf. This would have meant that 984 patients would have required support after discharge to ensure 7 days thromboprophylaxis. Eight per cent of patients $>75$ years old were from care homes, where staff may potentially administer LMWH. This left around 905 patients $\geq 85$ years old that may have required post-discharge thromboprophylaxis. This represents 2,442 district nurse visits per year (calculation not shown) to administer $\mathrm{LMWH}$, but this figure does not take into account any patient $<85$ years old who cannot self-administer $\mathrm{LMWH}$ or patients $\geq 85$ years old where a relative may be trained.

A $0.45 \mathrm{~L}$ sharps bin costs $£ 0.89$ (NHS supply chain website, http://my.supplychain.nhs.uk/Catalogue/product/fsl002 [Accessed 24 May 2019]) and dalteparin costs $£ 2.82$ per 5,000 unit syringe (the LMWH used in our local trust policy; British National Formulary Online, https://bnf.nice.org.uk/medicinal-forms/dalteparin-sodium. html [Accessed 25 May 2019]). In 2018, there were 5,532 medical discharges with a length of stay of 3-6 days. This would cost $£ 15,600.24$ per day in dalteparin post-discharge and $£ 4923.48$ in sharps bins. We estimated at least $16 \%$ of patients discharged on LMWH would need help with administration after discharge.

We performed an audit of medical patients with hospitalacquired thrombosis (HAT) who received less than 7 days prophylactic LMWH. From 01 May 2018 to 31 August 2018 there were 64 medical patients with HAT, of which only a minority of $14(22 \%)$ received less than 7 days thromboprophylaxis and that did not have a contraindication to LMWH. The median number of days of prophylaxis given in these 14 patients was 3.5; it is unclear what benefit an additional few days would make.
When the NICE recommendations were presented at the trust medicine board, there were concerns that discharges may be delayed to ensure the stipulated 7 days prophylactic LMWH.

There are limitations in our calculations, which have not been subject to formal analysis (including cost of HAT treatment). We support efforts to reduce HAT, however the guidance from NICE has potential burden of costs with benefit to a relative minority of patients. We would like to see further evidence in this area.

\section{Conflicts of interest}

Will Thomas has honorarium from Bayer and Pfizer and has previously worked on an advisory board for Daiichi-Sankyo.

Consultant haematologist, Cambridge University Hospitals NHS Foundation Trust, Cambridge, UK

TINA SLEEP

Anticoagulation specialist nurse, Cambridge University Hospitals NHS Foundation Trust, Cambridge, UK

AMY FORBES McNEIL

Anticoagulation specialist nurse, Cambridge University Hospitals NHS Foundation Trust, Cambridge, UK

STEPHEN WALLIS

Consultant physician, Cambridge University Hospitals NHS Foundation Trust, Cambridge, UK

\section{References}

1 National Institute for Health and Care Excellence. Venous thromboembolism in over 16s: reducing the risk of hospital-acquired deep vein thrombosis or pulmonary embolism. NICE Guideline [NG89]. London: NICE, 2018.

2 Shaprio S, Everington E, Roberts L, Ayra R. Venous thromboembolism. Clin Med 2019;19:262.

3 Lester W, Gomez K, Shapiro S et al. NICE NG89 recommendations for extended pharmacological thromboprophylaxis - is it justified and is it cost effective: a rebuttal from the British Society for Haematology. $\mathrm{Br}$ ] Haematol 2019 [published online ahead of print].

4 Stansby G, Donald I, Barry P, Dawoud D, Sharpin C. NICE NG89 recommendations for extended pharmacological thromboprophylaxis - is it justified and is it cost effective: a rebuttal from the British Society for Haematology - response to Lester et al. Br ] Haematol 2019 [published online ahead of print].

5 Rockwood K, Song X, Macknight C et al. A global clinical measure of fitness and frailty in elderly people. CMAJ 2005;173:489-95.

Address for correspondence: Dr Will Thomas, Haemophilia and Thrombophilia Centre, Cambridge University Hospitals NHS Foundation Trust, Hills Road, Cambridge CB2 OQQ, UK. Email: william.thomas@addenbrookes.nhs.uk 